\title{
Assessment of canopy vigor information from kiwifruit plants based on a digital surface model from unmanned aerial vehicle imagery
}

\author{
Jinru Xue ${ }^{1,2,3}$, Yeman Fan ${ }^{1,2,3}$, Baofeng $\mathrm{Su}^{1,2,3 *}$, Sigfredo Fuentes ${ }^{4}$ \\ (1. College of Mechanical and Electronic Engineering, Northwest A\&F University, Yangling 712100, Shaanxi, China; \\ 2. Key Laboratory of Agricultural Internet of Things, Ministry of Agriculture and Rural Affairs, Yangling 712100, Shaanxi, China; \\ 3. Shaanxi Key Laboratory of Agricultural Information Perception and Intelligent Services, Yangling 712100, Shaanxi, China; 4. School of \\ Agriculture and Food, Faculty of Veterinary and Agricultural Sciences, University of Melbourne, Parkville 3010. Victoria, Australia)
}

\begin{abstract}
Information about canopy vigor and growth are critical to assess the potential impacts of biotic or abiotic stresses on plant development. By implementing a Digital Surface Model (DSM) to imagery obtained using Unmanned Aerial Vehicles (UAV), it is possible to filter canopy information effectively based on height, which provides an efficient method to discriminate canopy from soil and lower vegetation such as weeds or cover crops. This paper describes a method based on the DSM to assess canopy growth (CG) as well as missing plants from a kiwifruit orchard on a plant-by-plant scale. The DSM was initially extracted from the overlapping RGB aerial imagery acquired over the kiwifruit orchard using the Structure from Motion (SfM) algorithm. An adaptive threshold algorithm was implemented using the height difference between soil/lower plants and kiwifruit canopies to identify plants and extract canopy information on a non-regular surface. Furthermore, a customized algorithm was developed to discriminate single kiwifruit plants automatically, which allowed the estimation of individual canopy cover fractions $\left(f_{c}\right)$. By applying differential $f_{c}$ thresholding, four categories of the CG were determined automatically: (i) missing plants; (ii) low vigor; (iii) moderate vigor; and (iv) vigorous. Results were validated by a detailed visual inspection on the ground, which rendered an overall accuracy of $89.5 \%$ for the method proposed to assess CG at the plant-by-plant level. Specifically, the accuracies for CG category (i)- (iv) were $94.1 \%, 85.1 \%, 86.7 \%$, and $88.0 \%$, respectively. The proposed method showed also to be appropriate to filter out weeds and other smaller non-plant materials which are extremely difficult to be distinguished by common colour thresholding or edge identification methods.
\end{abstract}

Keywords: canopy vigor, UAV imagery, digital surface model, kiwifruit plant, missing plants, photogrammetry, plant stress DOI: $10.25165 /$ j.ijabe.20191201.4634

Citation: Xue J R, Fan Y M, Su B F, Fuentes S. Assessment of canopy vigor information from kiwifruit plants based on a digital surface model from unmanned aerial vehicle 1magery. Int J Agric \& Biol Eng, 2019; 12(1): 165-171.

\section{Introduction}

Kiwifruit is an important cash value crop and their cultivation has become a major agricultural industry that promotes the economic development of the burgeoning kiwifruit producing regions in the northwest of China, the northeast of New Zealand, as well as Italy. However, traditional manual methods to manage a kiwifruit orchard are time-demanding and usually based on the monitoring of sentinel plants with extrapolation of management decisions without considerations of the natural spatial and temporal variability of orchards. Plant biotic and abiotic stresses can result in a partial or complete canopy and plant losses within an orchard. Furthermore, these effects can lead to significant economic losses depending on the severity of the specific detrimental effects which have a direct relationship with the production and quality of kiwifruits in the short and long term. Therefore, it is urgent to

Received date: 2018-09-03 Accepted date: 2018-12-18

Biographies: Jinru Xue, Master, research interests: precision agriculture, remote sensing of agriculture, Email: jinruxue@nwsuaf.edu.cn; Yeman Fan, Master, research interests: precision agriculture, agricultural aviation, Email: yemanfan@nwsuaf.edu.cn; Sigfredo Fuentes, PhD, Associate Professor, research interests: viticulture, remote sensing and plant physiology, Email: sfuentes@unimelb.edu.au.

*Corresponding author: Baofeng $\mathrm{Su}, \mathrm{PhD}$, Associate Professor, research interests: precision agriculture, remote sensing of agriculture. College of Mechanical and Electronic Engineering, Northwest A\&F University, Yangling 712100, Shaanxi, China. Tel: +86-15609279711, Email: bfs@ nwsuaf.edu.cn. develop fast, cost-effective and near real-time methods to obtain more accurate information from kiwifruit orchards that reflect accurately the spatial and temporal variability at the plant level for efficient decision making and crop management to maximize fruit quality and yield, especially in a changing climate.

Remote sensing technologies using different platforms, such as satellite, airborne and more recently Unmanned Aerial Systems (UAS), have been increasingly applied in the agricultural industry worldwide. Within the optical/visible domain (400-700 nm; RGB imagery), it offers a potential to extract spatial information from the canopy growth (CG) of kiwifruit plants in a non-destructive manner, with the objective of the development of decision support systems ${ }^{[1,2]}$. The UAS have been transformed nowadays in affordable and flexible solutions that can provide images at high spatial, temporal, and spectral resolution. UAS allows planning flights at a date that is very close to targeted phenological stages, allowing the detection of plant biotic, abiotic stresses or for pre-harvest characterization ${ }^{[3-5]}$. Furthermore, UAS has been one of the technologies with the highest rate of development and implementation for precision agriculture applications in recent years $^{[6,7]}$. In addition, most of the visible/near-infrared/thermal infrared indices that can be calculated from UAS remote sensing do not require atmospheric corrections, compared to satellite remote sensing ${ }^{[8]}$ due to low altitude surveys required (50-100 MAGL).

For kiwifruit orchards, higher spatial/temporal resolution based UAS remote sensing can provide a potential and efficient way to identify and measure CG. One of the main issues in row-planted 
crops is the individual plant characterization, which requires the isolation of pixels belonging to canopies from those belonging to soil or any other vegetation material such as weeds or cover crops. One common approach is to differentiate spectral properties between rows and inter-rows, which require multispectral cameras, it was first explored by applying a threshold on vegetation indices computed from multispectral images ${ }^{[9-11]}$ and, recently, machine learning algorithms ${ }^{[12,13]}$. The analysis based on spectral bands can result in some problems associated to the same plant material presenting different spectra (different growth level), separation of plant material and shade, different plant material with similar spectra (such as the case of weeds or cover crops in the inter-row) and mixed information per pixel such as soil/plant associated to the specific spatial resolution from the imagery ${ }^{[14]}$. Another approach is by recognizing the edges of the canopy using different edging algorithms applied to multispectral or infrared thermal imagery ${ }^{[15]}$. However, for multispectral, this method will encounter similar problems described before and for infrared thermal it could include in the analysis heat effect from soils and other objects (training wires and poles) specially in the edges of canopies. An alternative approach is a system based on plant shape and architecture, such as by the implementation of the DSM, which would discriminate more efficiently the regions of interest or plants through its canopy height, which are significantly different to ground and other objects height, such as grass, weeds, and cover crops.

Furthermore, UAS can be implemented to assess different physiological aspects of plants, such as: (1) vigor and canopy architecture monitoring (using multispectral cameras and NDVI) ${ }^{[16,17]}$; (2) plant water status (using infrared thermal cameras $)^{[18,19]}$ or both and iii) sub-meter evapotranspiration estimations $^{[20]}$. However, sub-meter evapotranspiration estimation systems from remote sensing data also require specialized personnel for aircraft operation, data acquisition and analysis. Some of these applications require higher instrumentation costs compared to high definition RGB cameras. Other studies, based on both the spatial and the spectral properties of crop rows, applied a Fourier transform to the red band of RGB images as a differentiation criteria ${ }^{[21]}$. However, these remote sensing methods for CG assessment are also based on multispectral remote sensing, which also includes the drawbacks mentioned before.

The kiwifruit plant is a vine-like plant that needs to be trained and planted in rows with fruits positioned at breast level for easy picking at harvest. A Digital Surface Model (DSM) is based on identifiable ground objects including buildings, forest trees and crop plants, among others, which can provide elevation information of various ground objects to show surface undulation conditions and crop growth conditions ${ }^{[22]}$. The DSM approach has been successfully applied to major $\mathrm{crops}^{[23]}$, for example, Adam et al. ${ }^{[24]}$, used the Structure from Motion (SfM) algorithm to model grapevine canopy structure in a vineyard site in the Texas Hill Country, and analyzed the data using a stepwise regression model to attempt to predict LAI, results showed a moderate $R^{2}$ value of 0.6. Grigorijs et al. ${ }^{[25]}$ used point clouds derived from SfM matching techniques obtained from UAS to detect individual trees, measured tree heights, and provided RGB estimates in Australian tropical savannas. Marie and Frédéric ${ }^{[11]}$ developed an algorithm for vineyard structural characteristic estimation based on dense point clouds derived from the RGB colour model images acquired with a UAV. Finally, Su et al. ${ }^{[22]}$ used DSM acquired from UAV RGB images to assess affected and missing grapevine canopies

affected by frost.

This work describes the development of a DSM for a kiwifruit orchard derived from the RGB colour model based images acquired using a UAV through three-dimensional (3D) reconstruction with SfM algorithm techniques. Furthermore, photogrammetry algorithms were applied to assess the CG at the plant-by-plant level to be finally classified into four classes of CG: (i) missing plants; (ii) low vigor; (iii) moderate vigor; and (iv) vigorous.

\section{Materials and methods}

\subsection{Experimental site}

The experimental field is located at the kiwifruit orchard belonging to Zhouzhi prefecture in the north Qinling Mountain Area of Shaanxi Province $\left(34.14^{\circ} \mathrm{N}, 108.09^{\circ} \mathrm{E}\right)$ (Figure 1). The climate of the region is classified as a temperate arid with a mean annual precipitation of about $180 \mathrm{~mm}$. The frost-free season corresponds to $200 \mathrm{~d}$ per year from April to October. The soil is mainly clay in texture with low water infiltration velocity. After many years of development, this area has become a renowned region for kiwifruit in China. The experimental kiwifruit orchard consisted in 5-year-old kiwifruit plants, cultivar Green kiwifruit, planted in a $\mathrm{S}-\mathrm{N}$ row direction with spacing between plants and between rows of $1.5 \mathrm{~m} \times 4.2 \mathrm{~m}$ respectively with a total of 1587 plants per hectare.

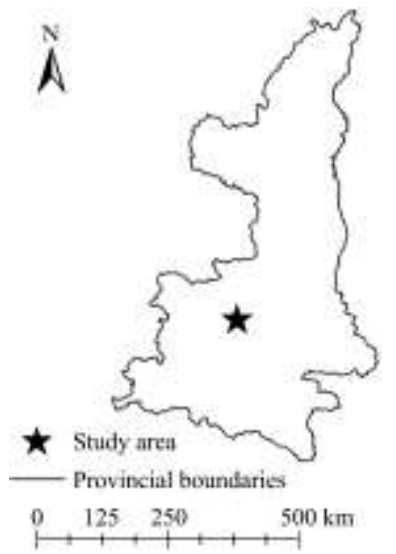

a. Experimental field located at Shaanxi region in China

Figure 1 Experimental orchard site

\subsection{UAV Imagery Acquisition}

In this study, a DJI Phantom 3 Vision plus quadcopter (SZ DJI Technology Co., Ltd., Shenzhen, China) was used as the survey platform. This aircraft is an affordable small sized four-axis quadrotor aerial vehicle with a flight control system, a three-axis stabilizing gimbal and WiFi communication capabilities. The quadcopter specifications are: $1280 \mathrm{~g}$ of weight, $4480 \mathrm{~mA} \cdot \mathrm{h}$ of battery capacity, a maximum speed of $15 \mathrm{~m} / \mathrm{s}$, and maximum flight time of around $25 \mathrm{~min}$. To reduce the complexity of the UAS operation, the Pix4Dcapture software (Pix4D, Lausanne, Switzerland) was used to control the flight and capture the RGB images. The Pix4Dcapture allows using the UAS as a mapping and measuring tool by defining autonomous mapping flights through pre-defined waypoints for data acquisition.

A parrot sequoia camera (Parrot, France) was mounted on the UAV to be used as the remote sensor (Figure 2). The camera specifications are: 16 MP RGB sensor, four narrowband and synchronized 1.2 MP monochrome sensors. The camera can be used to take pictures of agricultural fields in several spectral bands which measure the state of the vegetation: Green $(550 \mathrm{~nm}$ 
wavelength, $40 \mathrm{~nm}$ bandwidth), Red (660 $\mathrm{nm}$ wavelength, $40 \mathrm{~nm}$ bandwidth), Red Edge (735 nm wavelength, $10 \mathrm{~nm}$ bandwidth) and Near Infrared (790 nm wavelength, $40 \mathrm{~nm}$ bandwidth). It is an automatically calibrated camera because of the sunshine module and has an integrated GPS/GNSS to locate the camera when photos are being taken. In addition, users can connect a smart mobile device wirelessly to the UAV and the remote camera via WiFi to control the drone and to obtain real-time transmission of images and videos. Remote images were acquired through UAV fights that were performed on May 16th, 2017 during the kiwifruit growing period, the images were recorded on a flash card, allowing $60 \%$ to $80 \%$ overlapping along and between the tracks, according to the nominal $25.2 \mathrm{~km} / \mathrm{h}$ UAV flight speed at $40 \mathrm{~m}$ flight altitude. For this study, only the RGB bands were considered, which can be accessed from any ubiquitous digital camera.

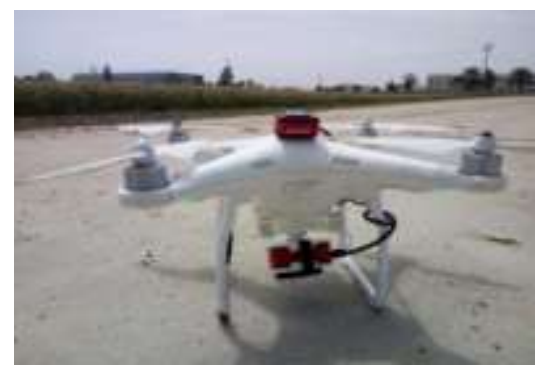

Figure 2 DJI Phantom 3 Vision+ UAV remote sensing platform used for this study with the parrot sequoia camera mounted on

\subsection{Geospatial analysis}

The SfM technique facilitates the establishment of DSM and consists in a digital reconstruction technique, which is overlapped are extracted from a series of images captured from different angles of view to achieve a $3 \mathrm{D}$ reconstruction according to the image feature matching algorithm ${ }^{[17]}$. With the camera parameters and 3D data, the SfM technique can be used to perform motion estimation according to the geometric relationships between 2D images of multiple view angles. Then the SfM optimizes the motion calculation and determines $3 \mathrm{D}$ points with bundle adjustment to finally obtain a dense 3D point cloud through point cloud extension ${ }^{[13]}$. Finally, the DSM was built on the basis of the estimated camera position and the images themselves. In this research, the Pix4Dmapper Professional software was used for this task, Pix4Dmapper is a 3D reconstruction software based on the principle of SfM and the mosaicking process was fully automated involved the following phases: (1) Initial Processing, (2) Point Cloud and Mesh, (3) DSM, Orthomosaic and Indexing. After feature extraction and matching of UAS aerial images, a 3D point cloud was generated and the DSMs were obtained in Tag Image File Format (TIFF). To extract the planting pattern and characteristics of kiwifruit orchard, the GIS software ArcGIS (Esri, Inc., Redlands, UAS) was used for georeferencing and crop the DSM for the selected study site.

\subsection{Description of the kiwifruit CG assessment method}

Figure 3 presents an overview of the algorithms and flow chart used to extract CG information from the kiwifruit orchard. The whole analysis process start with the image binarization from the overlapping RGB image acquired with the UAS (Figure 3, Step A). This is achieved by obtaining the DSM with the Pix4Dmapper software and then applying an adaptive threshold algorithm to extract plant positions and to filter weed and soil background. Then, RGB image is rotated and cropped to adjust planting row to a horizontal position to establish anchor points and masks as marks for further assessment (Figure 3, Step B). Finally, the canopy vigor of the whole kiwi orchard is assessed (Figure 3, Step C), firstly, the binary image is rotated, cropped and generating reference points and masks with the same position as anchor points and masks in the RGB image to calculate canopy cover fraction $\left(f_{c}\right)$ Then, every single mask is judged according to the specific threshold value.

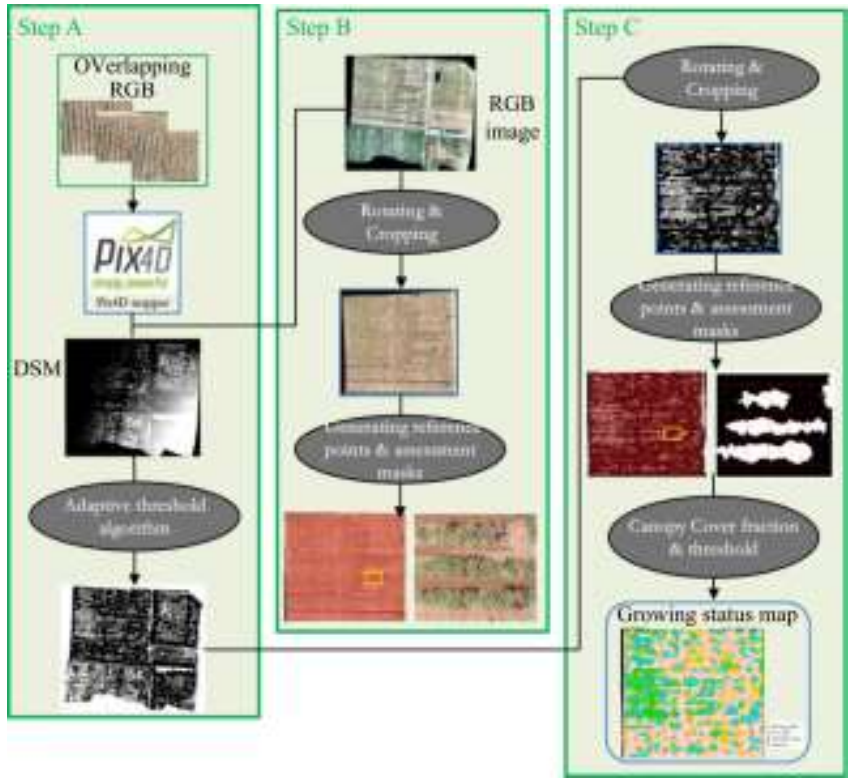

Figure 3 Assessment of kiwifruit orchard showing the different steps to characterize and filter canopies from background information (Step A) and to classify UAV at the plant-per-plant scale (Step B and Step C)

\section{Results and discussion}

\subsection{Adaptive threshold algorithm to obtain plant position (Step A)}

Figure 3 shows the DSM generated from the RGB images obtained with the UAS. The highest point for discrimination within the research region was $2 \mathrm{~m}$ above the ground. There was a distinctive difference in height between the kiwifruit trees (white stripped area) compared to objects in the inter-row. The thresholding method was then exported as a binary image. At this stage, the DSM allows only qualitative analysis of canopies height in relation to ground, therefore, the area corresponding to missing plant cannot be assessed directly via the DSM of study region because of the complicated surface structures of the undulating ground in the kiwifruit orchard. Figure 4 also shows the kiwifruit trees and their distribution in the non-regular or sloped fields, such as those encountered in the trial site. This presented a problem since a common discriminating threshold will over or underestimate the real height of the whole canopy (i.e. Line1, Line2, and Line3 corresponding to three different thresholds).

As a result, a local threshold method was implemented, the adaptive threshold algorithm, which was denoted as $T(x, y)$ was implemented by setting a subset of pixels in a square area $\Omega_{x, y}$ of size $\mathrm{I}_{\mathrm{w}}$ to calculate the local threshold by subtracting a constant $\mathrm{C}$ obtained from the average elevation of the neighborhood pixels from the pixel point $(x, y)$. The local thresholds are then used to filter the kiwifruit tree from the background, the constant $C$ is set to extract canopies which are lower than the average height in the sliding window. The size of $I_{w}$ and the value of $C$ was confirmed according to the actual situation of the experiment. Using $\operatorname{src}(x, y)$ to represent the pixel value from each point in the original image, $\operatorname{dst}(x, y)$ represent the value of the pixel in the resulting binary 
image, with the binarization process represented mathematically by Equation (1).

$$
\operatorname{dst}(x, y)=\left\{\begin{array}{l}
1, \text { if } \operatorname{src}(x, y)>T(x, y) \\
0, \text { if } \operatorname{src}(x, y)>T(x, y)
\end{array}\right.
$$
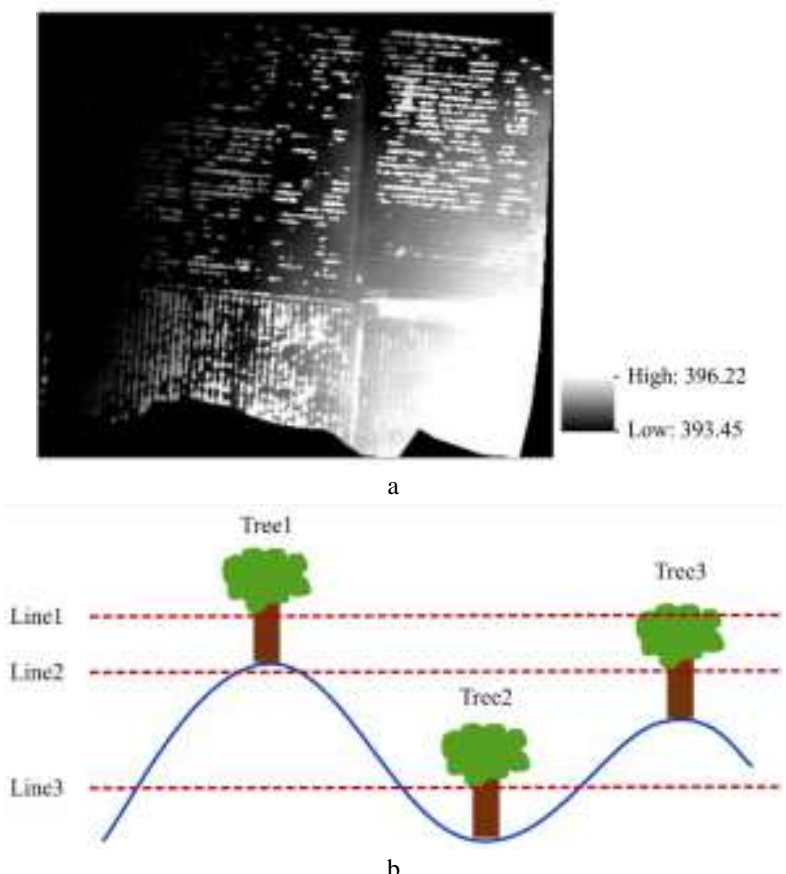

b

Figure 4 (a) DSM extracted from the of kiwifruit orchard; (b) Diagram representing terrain slope changes within the orchard and the effect on a fixed height thresholding

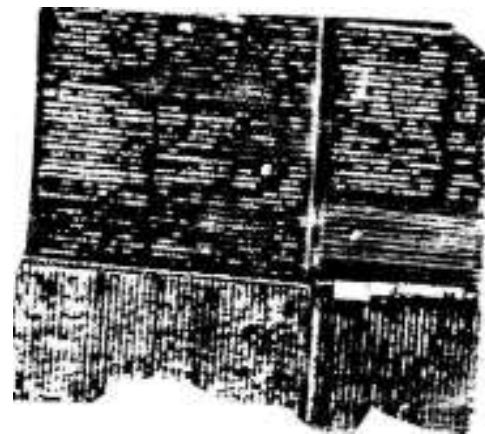

Figure 5 Resulting binary image obtained by using the adaptive threshold algorithm for height selection for a kiwifruit orchard studied corresponding to the polygon in blue

As shown in Figure 5, the binary image obtained after the application of an adaptive thresholding algorithm for height selection contains all the boundaries from kiwifruit plants. This method can also remove the gradient background effectively and extract kiwifruit plants in non-uniform or slope ground conditions.

3.2 Establishment of reference points and assessment masks to separate canopy information from the inter-row material (Step B)

The homogeneous plantation pattern presented in this study can be used to separate the information from the processed image form the kiwifruit orchard in a plant-by-plant scale. Therefore, individual kiwifruit canopies can be detected by creating a set of reference points by linear interpolation, each point corresponding to a detection area of interest corresponding to a whole kiwifruit canopy.

Based on this principle, the initial row positions and end points of 12 sampled rows (red crosses; Figure 6a) were assigned in the RGB image and were used to calculate the row orientation, the assigned rows were selected at intervals of two rows(with a total of 36 rows). Using $\theta$ to represents the orientation between the kiwifruit planting row and the image $X$ coordinate axis, $x_{1}$ and $x_{2}$ are the horizontal components and $y_{1}$ and $y_{2}$ are the vertical component of the verges of rows, then the orientation from each selected row can be computed by Equation $(2)^{[26]}$.

$$
\theta=\tan ^{-1}\left(\frac{\left|y_{2}-y_{1}\right|}{\left|x_{2}-x_{1}\right|}\right) \cdot\left(\frac{180}{\pi}\right)
$$

The orientation from the west-east of linear features (i.e., rows in this case) was given by $\theta$. The average angle of the 12 rows obtained is shown in Table 1. Once the averaged angle of the rows was calculated, the image was rotated by the averaged $\theta$ (Figure 6b)

Table 1 Line orientation corresponding to each kiwifruit

\begin{tabular}{|c|c|c|c|c|c|c|}
\hline Row & 2 & 5 & 8 & 11 & 14 & 17 \\
\hline$\theta /\left(^{\circ}\right)$ & 5.33 & 5.26 & 4.56 & 4.78 & 5.03 & 5.17 \\
\hline Row & 20 & 23 & 26 & 29 & 32 & 35 \\
\hline$\theta /\left(^{\circ}\right)$ & 5.06 & 4.88 & 5.18 & 5.24 & 5.15 & 5.28 \\
\hline \multicolumn{2}{|c|}{ Average } & & & 5.07 & & \\
\hline
\end{tabular}
planting lines and the average angle of rows

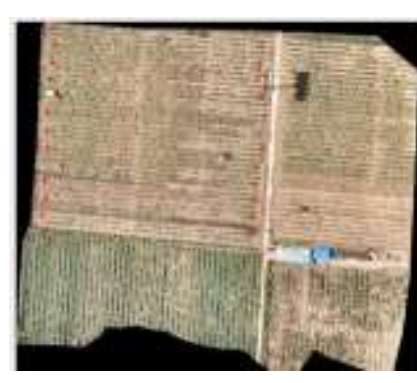

a

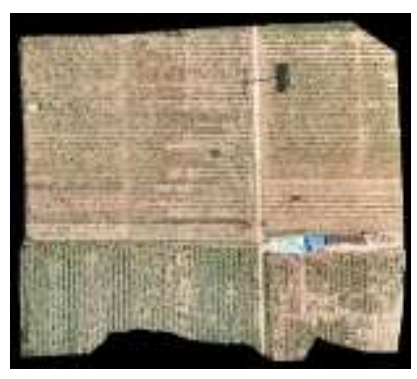

b
Figure 6 (a) Selected kiwifruit planting rows (two verges from each row marked with red crosses); (b) Rotated RGB image of kiwifruit orchard

The rotated image requires the creation of reference points of interest to identify individual plants. The reference points refer to stem position from plants or the centroid of the designated planting area. In order to achieve this, it was necessary to combine the known data of planting density for rows and inter-row distances of $1.5 \mathrm{~m} \times 4.2 \mathrm{~m}$ respectively. With this information, it is possible to automatically obtain the number of rows and the kiwifruit trees cultivated in each row, which corresponded to 36 and 100, respectively. As a result, the pixel position corresponding to the centroids can be computed by the pixel interval of 36 rows and 100 points per row through linear interpolation to generate points and masks automatically. The rotated image (Figure 6b) was then used to calculate automatically the position of areas corresponding to individual plants that allows the calculation and storage of any information related to pixel count or channel (RGB) data using programming loops (Figures 7a and 7b).

\subsection{Binary image generation to assess of kiwifruit CG (Step} C)

Reference points and masks with the same position as RGB image were then set in the binary image. From each sub-image corresponding to individual kiwifruit plants a $f_{c}$ can be calculated automatically, extracted and stored as pixels of canopy over total pixels of the sub-image. Figure $7 \mathrm{a}$ shows the resulting binary image of the kiwifruit orchard study area, which can be used as a mask to be overlapped with the processed image to obtain specific plant-by-plant areas or RGB information. Figure $8 \mathrm{~b}$ shows the 
combined masked image and processed image (left), from which individual plant analysis can be performed to calculate $f_{c}$ (right).

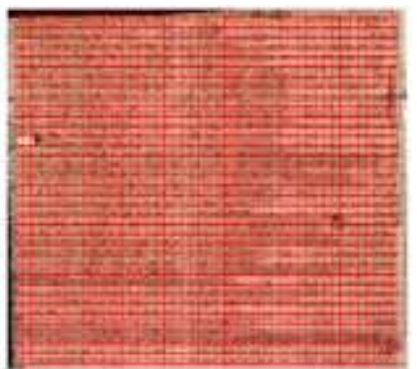

a

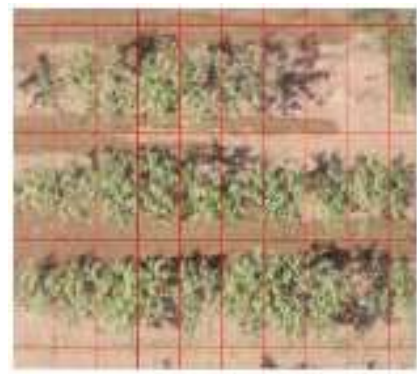

b
Figure 7 (a) Reference points and assessment masks obtained by linear interpolation to extract information of single kiwifruit trees automatically from either binary or RGB images. (b) Close up from a region that shows clearly the area per plant with centroids identified automatically (marked as *)

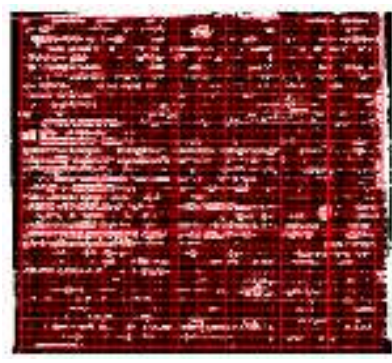

a

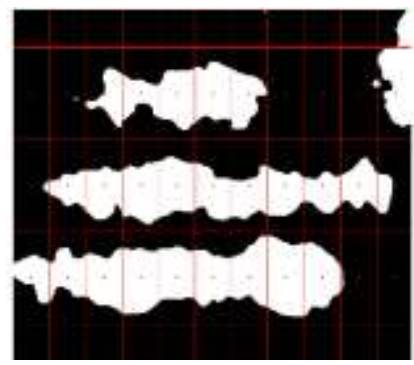

b
Figure 8 (a) Combined binary and RGB image of the kiwifruit orchard studied for matrix analysis using the customized code written in Matlab®; (b) Reference points and assessment masks obtained by linear interpolation to isolate single kiwifruit plants for $f_{c}$ automated analysis from the binary image

To determine the specific threshold values for CG classification, 200 sub-images were randomly selected to analyse their specific $f_{c}$ values (Figure 9).

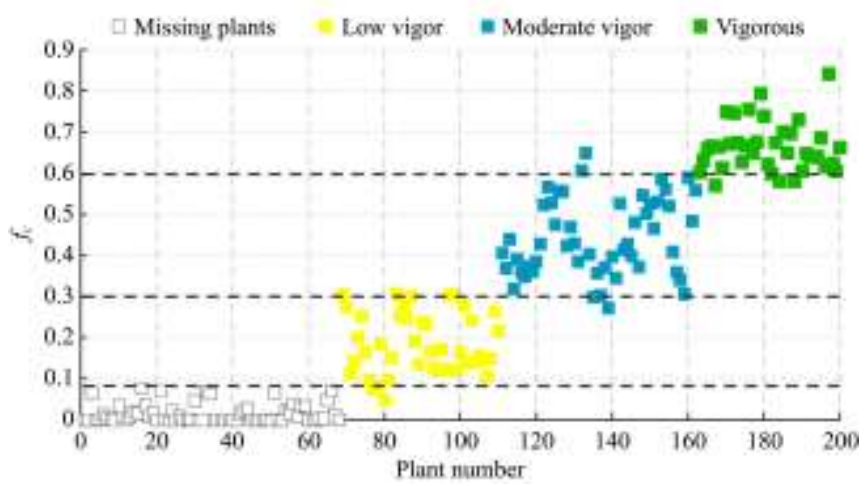

Figure $9 f_{c}$ from 200 random kiwifruit plants locations within the studied orchard and separated into four thresholds: (i) missing plant (white filled boxes) $f_{c} \leq 0.08$; (ii) low vigor (yellow filled boxes) $0.08<f_{c} \leq 0.3$; (iii) moderate vigor bright blue filled boxes) $0.3<$ $f_{c} \leq 0.6$ and (iv) vigorous (green filled boxes) $f_{c}>0.6$

The canopy vigor classification was quantified as i) missing plants using the criteria based on inexistent o minimal canopy pixels encountered in the specific analyzed region $\left(f_{c} \leq 0.08\right)$, ii) low vigor $\left(0.08<f_{c} \leq 0.3\right)$, iii) moderate vigor $\left(0.3<f_{c} \leq 0.6\right)$ and iv)vigorous $\left.\left(f_{c}>0.6\right)\right)$. The missing plant criteria was based on an extremely low level of vegetation, the low vigor trees related to plants that are partly affected by biotic/abiotic factors, the moderate vigor level was related to well-growing condition and less affected canopies, while the vigorous level corresponded to high vegetative growth that can be related to overgrowth or a dense biomass, which can be a result from over-irrigation or fertilization.

Using these criteria and thresholds, the sub-images were classified by colour automatically (Figure 10) as: (i) missing plants (white filling corresponding to 1517 plants or $42.14 \%$ of total plants); (ii) low vigor (yellow filling corresponding to 713 plants or $19.81 \%$ of total plants); (iii) moderate vigor (bright blue filling corresponding to 992 plants or $27.56 \%$ of total plants); and (iv) vigorous trees (green filling corresponding to 378 plants or $10.50 \%$ of total plants). Most of the missing kiwifruit plants in white filled boxes were surrounded by affected kiwifruit trees (low vigor), which makes sense if they were affected by biotic/abiotic stresses. On the contrary, vigorous canopies in green filled boxes are surrounded by the vigorous canopy and moderate vigor canopy gathered in the middle part of studied kiwifruit orchard.

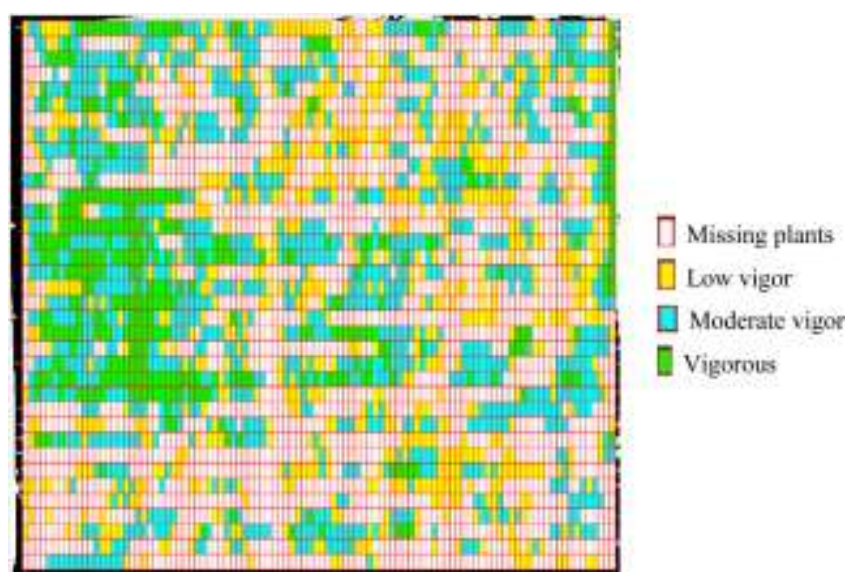

Figure 10 Automated classification of kiwifruit growing status level per plant, missing plants (white filled boxes): $f_{c} \leq 0.08$, low vigor (yellow filled boxes): $0.08<f_{c} \leq 0.3$, moderate vigor (bright blue filled boxes): $0.3<f_{c} \leq 0.6$, and ArcGIS vigorous (green filled boxes): $f_{c}>0.6$

The accuracy test for the identification and classification method proposed was evaluated by comparing results using the algorithms proposed against the real growing condition obtained by visual inspection of expertise from the study area. Results showed high accuracy in the estimation of canopy cover by the method proposed with $88.0 \%$ correct identification of vigorous, $86.7 \%$ of moderate vigor, $85.1 \%$ of low vigor, $94.1 \%$ of missing plants and an overall accuracy of the method of $89.5 \%$ (Table 2). These results can be also applied to improve the accuracy of the DSM method implemented to separate canopies from the background, since over and underestimations of canopy cover were minimized.

Table 2 Comparison of the performance of proposed algorithm (Estimated) with visual inspection (Observed)

\begin{tabular}{lcccccc}
\hline \multirow{2}{*}{ Observed } & \multicolumn{6}{c}{ Estimated } \\
\cline { 2 - 7 } & Vigorous & $\begin{array}{c}\text { Low } \\
\text { vigor }\end{array}$ & $\begin{array}{c}\text { Moderate } \\
\text { vigor }\end{array}$ & $\begin{array}{c}\text { Missing } \\
\text { plants }\end{array}$ & Overall & Accuracy \\
\hline Vigorous & 22 & 1 & 0 & 2 & 25 & $88.0 \%$ \\
Low vigor & 1 & 53 & 1 & 5 & 60 & $86.7 \%$ \\
Moderate vigor & 1 & 2 & 40 & 4 & 47 & $85.1 \%$ \\
Missing plants & 0 & 0 & 4 & 64 & 68 & $94.1 \%$ \\
Overall & 24 & 56 & 45 & 75 & 200 & $89.5 \%$ \\
\hline
\end{tabular}

It is important to note that the method proposed produces an automatic classification of vigor of kiwifruit plants in a map form, 
but also the classification is numerical. Therefore, growers can have the specific row and plant with the respective classification on a list, which could help significantly to perform targeted managements for plant replacement, canopy management, fertilization and irrigation at a plant-by-plant scale. This method can also be used to obtain other canopy architecture parameters, such as leaf area index (LAI) and canopy porosity $(\phi)$ using the same algorithms for cover photography and computer application (VitiCanopy) developed by De et al. ${ }^{[27]}$, Arachchige et al. ${ }^{[28]}$ also used 'Canny edge detection' and 'Otsu's' methods to derive canopy porosity to quantifying the severity of phytophthora root rot disease using RGB digital photographs.

\section{Conclusions}

A method for automated assessment of canopy vigor for a kiwifruit orchard using DSM from Unmanned Aerial Imagery was presented. The proposed method effectively reduced the complexity of background information in kiwifruit orchard by filtering topography using an adaptive threshold algorithm and accurately extracts kiwifruit rows. Conversely to methods based on classification of multispectral or RGB images, the method proposed in this paper is not sensitive to the presence of weed between the rows.

Since the method proposed produces an automatic classification of vigor of kiwifruit plants in a map form, but also the classification is numerical, growers can have the specific row and plant with the respective classification on a list to target management practices. Other canopy architecture parameters, such as leaf area index (LAI) and canopy porosity $(\phi)$ can also be obtained using the same algorithms for cover photography and computer application.

However, the accuracy of the results can be affected by the quality of Unmanned Aerial Imagery, since the quality of DSM rest with the flight condition such as UAV speed, camera resolution, field of view and wind. Besides, the threshold of $f_{c}$ need to adjust when implementing the method in other orchard because of the difference between rows and inter-row, as well as the canopy size.

\section{Acknowledgements}

This study was supported by the National Key Research and Development Program of China (No. 2017YFD0700402), the Key Science and Technology Program of Shaanxi Province, China (No. S2016YFNY0066), the Scientific Research Foundation for the Returned Overseas Chinese Scholars, State Education Ministry; Part of this research was supported by the Digital Viticulture program funded by the University of Melbourne's Networked Society Institute, Australia.

\section{[References]}

[1] Hall A, Lamb D, Holzapfel B, Louis J. Optical remote sensing applications in viticulture: A review. Australian Journal of Grape and Wine Research, 2002; 8(1): 36-47.

[2] Pinter Jr P J, Hatfield J L, Schepers J S, Barnes E M, Moran M S, Daughtry $\mathrm{C} \mathrm{S}$, et al. Remote sensing for crop management. Photogrammetric Engineering \& Remote Sensing, 2003; 69(6): 647-64.

[3] Torres-Sánchez J, López-Granados F, de Castro A I, Peña-Barragán J M. Configuration and specifications of an unmanned aerial vehicle (UAV) for early site specific weed management. PloS One, 2013; 8(3): e58210.

[4] Hunt E R, Cavigelli M, Daughtry C S, Mcmurtrey J E, Walthall C L. Evaluation of digital photography from model aircraft for remote sensing of crop biomass and nitrogen status. Precision Agriculture, 2005; 6(4):
359-378.

[5] Schirrmann M, Giebel A, Gleiniger F, Pflanz M, Lentschke J, Dammer $\mathrm{K}-\mathrm{H}$. Monitoring agronomic parameters of winter wheat crops with low-cost UAV imagery. Remote Sensing, 2016; 8(9): 706.

[6] Shamshiri R R, Hameed I A. Fundamental research on unmanned aerial vehicles to support precision agriculture in oil palm plantations. Agricultural Robots-Fundamentals and Application, 2018.

[7] Lan Y B, Shengde C, Fritz B K. Current status and future trends of precision agricultural aviation technologies. Int J Agric \& Biol Eng, 2017 10(3): $1-17$

[8] Hadjimitsis D G, Papadavid G, Agapiou A, Themistocleous K, Hadjimitsis $\mathrm{M}$, Retalis A, et al. Atmospheric correction for satellite remotely sensed data intended for agricultural applications: impact on vegetation indices. Natural Hazards and Earth System Sciences, 2010; 10(1): 89-95.

[9] Puletti N, Perria R, Storchi P. Unsupervised classification of very high remotely sensed images for grapevine rows detection. European Journal of Remote Sensing, 2014; 47(1): 45-54.

[10] Candiago S, Remondino F, de Giglio M, Dubbini M, Gattelli M. Evaluating multispectral images and vegetation indices for precision farming applications from UAV images. Remote Sensing, 2015; 7(4): 4026-4047.

[11] Weiss M, Baret F. Using 3D point clouds derived from UAV RGB imagery to describe vineyard 3D Macro-structure. Remote Sensing, 2017 9(2): 111.

[12] Poblete-Echeverría C, Olmedo G F, Ingram B, Bardeen M. Detection and segmentation of vine canopy in ultra-high spatial resolution RGB imagery obtained from unmanned aerial vehicle (UAV): A case study in a commercial vineyard. Remote Sensing, 2017; 9(3): 268.

[13] Poblete T, Ortega-Farías S, Moreno M A, Bardeen M. Artificial neural network to predict vine water status spatial variability using multispectral information obtained from an unmanned aerial vehicle (UAV). Sensors, 2017; 17(11): 2488.

[14] Fuentes S, Poblete-Echeverría C, Ortega-Farias S, Tyerman S, De Bei R. Automated estimation of leaf area index from grapevine canopies using cover photography, video and computational analysis methods. Australian Journal of Grape and Wine Research, 2014; 20(3): 465-473.

[15] Park S, Ryu D, Fuentes S, Chung H, Hernández-Montes E, O’Connell M. Adaptive estimation of crop water stress in nectarine and peach orchards using high-resolution imagery from an unmanned aerial vehicle (UAV). Remote Sensing, 2017; 9(8): 828.

[16] Di Gennaro S F, Rizza F, Badeck F W, Berton A, Delbono S, Gioli B, et al. UAV-based high-throughput phenotyping to discriminate barley vigour with visible and near-infrared vegetation indices. International Journal of Remote Sensing, 2017: 1-15.

[17] Duan T, Chapman S, Guo Y, Zheng B. Dynamic monitoring of NDVI in wheat agronomy and breeding trials using an unmanned aerial vehicle. Field Crops Research, 2017; 210: 71-80.

[18] Poblete-Echeverría C, Sepulveda-Reyes D, Ortega-Farias S, Zuñiga M, Fuentes S, editors. Plant water stress detection based on aerial and terrestrial infrared thermography: a study case from vineyard and olive orchard. XXIX International Horticultural Congress on Horticulture: Sustaining Lives, Livelihoods and Landscapes (IHC2014): 1112; 2014.

[19] Zia S, Du W, Spreer W, Spohrer K, He X, Muller J. Assessing crop water stress of winter wheat by thermography under different irrigation regimes in North China Plain. Int J Agric \& Biol Eng, 2012; 5(3): 24-34.

[20] Kustas W P, Anderson M C, Alfieri J G, Knipper K, Torres-Rua A, Parry C $\mathrm{K}$, et al. The grape remote sensing atmospheric profile and evapotranspiration experiment (GRAPEX). Bulletin of the American Meteorological Society, 2018.

[21] Chanussot J, Bas P, Bombrun L, editors. Airborne remote sensing of vineyards for the detection of dead vine trees. Geoscience and Remote Sensing Symposium, 2005 IGARSS'05 Proceedings 2005 IEEE International, IEEE, 2005.

[22] Su B F, Xue J R, Xie C Y, Fang Y L, Song Y Y, Fuentes S. Digital surface model applied to unmanned aerial vehicle based photogrammetry to assess potential biotic or abiotic effects on grapevine canopies. Int $\mathbf{J}$ Agric \& Biol Eng, 2016; 9(6): 119-130.

[23] Bendig J, Bolten A, Bareth G. UAV-based imaging for multi-temporal very high resolution crop surface models to monitor crop growth variability. Photogrammetrie-Fernerkundung-Geoinformation, 2013; 6(6): 551-562.

[24] Mathews A J, Jensen J L. Visualizing and quantifying vineyard canopy 
LAI using an unmanned aerial vehicle (UAV) collected high density structure from motion point cloud. Remote Sensing, 2013; 5(5): 2164-2183.

[25] Goldbergs G, Maier S W, Levick S R, Edwards A. Efficiency of individual tree detection approaches based on light-weight and low-cost UAS imagery in Australian Savannas. Remote Sensing, 2018; 10(2): 161.

[26] Nolan A, Park S, Fuentes S, Ryu D, Chung H, editors. Automated detection and segmentation of vine rows using high resolution UAS imagery in a commercial vineyard. Proceedings of the 21st International Congress on Modelling and Simulation, Gold Coast, Australia; 2015.

[27] De Bei R, Fuentes S, Gilliham M, Tyerman S, Edwards E, Bianchini N, et al. VitiCanopy: A free computer APP to estimate canopy vigor and porosity for grapevine. Sensors, 2016; 16(4): 585.

[28] Salgadoe S, Robson A J. Quantifying the severity of phytophthora root rot disease in avocado trees using image analysis. Remote Sensing, 2018; 10(2). DOI: 10.3390/rs10020226. 